\title{
Circumnuclear Disks in Early-Type Galaxies as Evidence for Secondary Formation Events
}

\author{
Sil'chenko Olga K. \\ Sternberg Astronomical Institute, University av. 13, Moscow 119899, \\ Russia; olga@sai.msu.su
}

The phenomenon of chemically distinct galactic nuclei is quite new and hardly studied yet. During the last 10 years we search for chemically distinct nuclei in disk galaxies, and after our first work (Silchenko, Afanasiev, \& Vlasyuk 1992) two dozens of those are already found. A question posed now is if the galaxies with chemically distinct nuclei have also distinct circumnuclear disks and if these disks can be identified with chemically distinct areas. An answer for the first part of the question is certainly 'YES'; the second part is far less clear. The observations presented here are made during 1996-1998, mostly with the Multi-Pupil Field Spectrograph (MPFS) of the $6 \mathrm{~m}$ telescope (Afanasiev et al. 1990). The MPFS allows to obtain a set of 96 to 240 spectra from an extended area; each spectrum corresponds to an element of $1.3^{\prime \prime} \times 1.3^{\prime \prime}$ before mid-1998 and $1.0^{\prime \prime} \times 1.0^{\prime \prime}$ later. Seeings were $1 . .5-2 ! 5$. What do we mean when telling about chemically distinct nuclei? We recognize them by a sharp drop of metal absorption-line equivalent widths when passing from the point-like nuclei to the surrounding bulges. Figure 1 presents 4 examples of the radial profiles of the magnesium and iron absorption-line indices in the Lick system (Worthey et al. 1994). The data for M 31 are long-slit data from the $1 \mathrm{~m}$ telescope of the Special Astrophysical Observatory; the cross-sections are taken in 5 various position angles (for details, Silchenko, Burenkov, \& Vlasyuk 1998). The other data are azimuthally averaged measurements from the MPFS (for details, see Silchenko 1999a,b and Afanasiev \& Silchenko 2000). All the profiles decrease prominently within the radius comparable to the spatial resolution; in the bulges they flatten off. The metallicity drops between the nuclei and the bulges by a factor of 2 to 10 . The behaviours of the magnesium and iron indices can be similar or different. The most interesting feature is revealed by comparing the iron and magnesium profiles in NGC 1023 and NGC 7331: the magnesium peaks in the very center whereas the maxima of the iron index are at the $R \approx 1^{\prime \prime}$. We should treat this phenomenon as an evidence for a short-duration star formation burst in the center and a more prolongated one in its neighborhood.

Figure 2 presents radial variations of isophote ellipticity and fourth cosine Fourier coefficient for two of these galaxies. One can see a prominent maximum of $a_{4}$, ranging from +1.0 to +4.0 , within the radius of the fast metal-line index decrease. It is a sign of embedded circumnuclear stellar disk highly inclined. The corresponding maxima of ellipticity are also seen. However a typical radius of the circumnuclear disks, several tens of parsecs, is of order of one arcsecond in the most galaxies under consideration that can be resolved in photometric data from the HST but is hardly resolved in our observations with the MPFS. Therefore with our present knowledge in most cases we cannot unambiguously relate the chemically distinct cores and the circumnuclear stellar disks. 

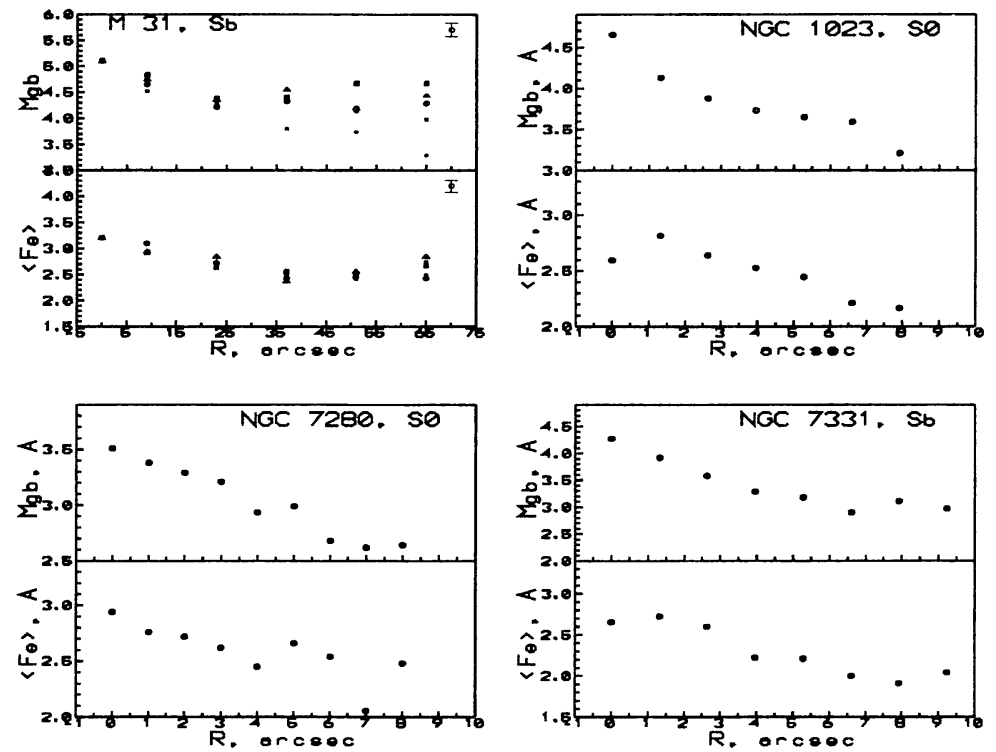

Figure 1. Radial profiles of absorption-line indices $\mathrm{Mgb}$ and $\langle\mathrm{Fe}\rangle$ for selected galaxies

\section{References}

Afanasiev, V.L. et al. 1990, Preprint SAO, N54

Afanasiev, V.L., \& Sil'chenko, O.K. 2000, AJ, 119, 126

Sil'chenko, O.K., Afanasiev, V.L., \& Vlasyuk, V.V. 1992, AZh, 69, 1121

Sil'chenko, O.K., Burenkov, A.N., \& Vlasyuk, V.V. 1998, A\&A, 337, 349

Sil'chenko, O.K. 1999a, AJ, 117, 2725

Sil'chenko, O.K. 1999b, AJ, 118, 186

Worthey, G., Faber, S.M., Gonzalez, J.J., \& Burstein, D. 1994, ApJS, 94, 687
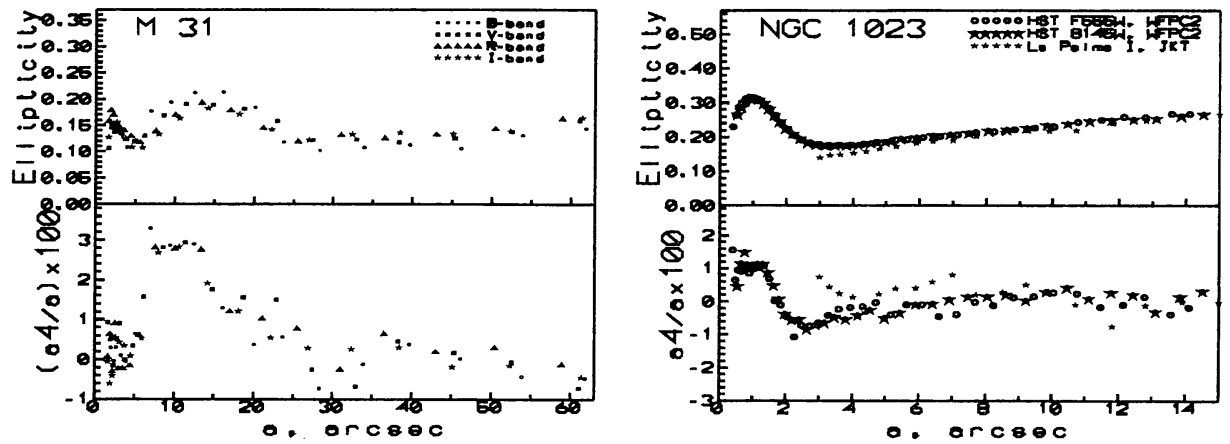

Figure 2. Radial profiles of isophote characteristics for selected galaxies 